\title{
Quantic Analysis of Formation of a Biomaterial of Latex, Retinol, and Chitosan for Biomedical Applications
}

\author{
Karina García-Aguilar ${ }^{1,2}$, Iliana Herrera-Cantú1 ${ }^{1}$, Erick Pedraza-Gress ${ }^{1}$, Lillhian Arely
} Flores-Gonzalez ${ }^{1}$, Manuel Aparicio-Razo ${ }^{1,4}$, Oscar Sánchez-Parada ${ }^{3}$, Emmanuel Vázquez-López ${ }^{1}$, Juan Jesús García-Mar ${ }^{1}$ and Manuel González-Pérez ${ }^{1,5}$

${ }^{1}$ Universidad Popular Autónoma del Estado de Puebla A.C. (UPAEP). Centro Interdisciplinario De Posgrados (CIP). Posgrado en Ciencias de la Ingeniería Biomédica.

${ }^{2}$ Instituto Tecnológico Superior de Coatzacoalcos. Académica de Ingeniería Bioquímica

${ }^{3}$ Escuela de Medicina Universidad Popular Autónoma del Estado de Puebla

${ }^{4}$ Benemérita Universidad Autónoma de Puebla, Facultad de Ciencias de la Electrónica

${ }^{5}$ Sistema Nacional De Investigadores. Nivel 1.

\begin{abstract}
The present work shows the quantum theoretical analysis and practical tests for the formation of a homogeneous mixture with Latex (Lx), Chitosan (Qn) and Retinol (Rl), which work as possible biomaterial for regeneration of epithelial tissue. Lx, Qn, and Rl compounds molecules were designed through Hyperchem to get the coefficient of electrostatic potential calculations. The amounts used to create the biomaterial are minimum depending on the quantities of molecules used in chemical design. A positive calculation was obtained for the reaction of these three compounds and the formation of the biomaterial in physical checking theory etc.
\end{abstract}

Keywords- Quantum analysis, Latex, Chitosan, Retinol, Biomaterial.

\section{INTRODUCTION}

Latex Lx is a colloidal aqueous suspension composed of fats, waxes and gummy resins, white and milky in appearance. Rubber (CA) is a hydrocarbon that is suspended in the Lx of trees in tropical and subtropical areas. [1] The CA or rubber is inside the Lx and presents a chemical formula c5H8. The chemical composition of the Lx has oils, sugars, mineral salts, proteins, terpenes, nucleic acids, hydrocarbons, waxes, resins, starch, tannins and balms. It has a neutral $\mathrm{pH}$ (7-7.2). Passed between twelve and twenty-four hours since removal, $\mathrm{pH}$ drops to 5.0 , came the coagulation of the substance when it is located with a pH equal to or less than 4.2. [2]

It is characterized for being a substance insoluble in water, electric and elastic resistance. Most of the Lx is commercial use is extracted from the Syrinx (Hevea brasiliensis) and is dedicated to the production of rubber.
The Lx prevents the entry of pathogenic micro-organisms and promotes the healing process when there is a wound. Allergen Lx Hev 2 b with properties $\beta$-1, 3-glucanase accelerate the hydrolytic scission of polymers $\beta-1,3$ glucans, basic component of the cell wall of fungi. This protein can prevent fungal infections to the plant through the degradation of the cell wall of fungal pathogens. The allergen in latex Hev b 11 endoquitinasa activity and could participate in the hydrolytic scission of chitin. Another protein that plays a role in defense is the hevamina. Acts by catalyzing the scission of the links $\beta-1$, 4-glucosidic linkages of chitin and the peptidoglycans of cell surface. [3]

Studies with Lx only explain their function with patients who have diabetic ulcers. The idea was to implement a template for a biomaterial ( $\mathrm{Lx}$ ) for the control of the pressure of the diabetic foot. [4]

Chitosan (QN) is a polysaccharide that is naturally in the cell walls of some fungi. The main source of production is between alkaline chitin hydrolysis. The presence of groups amines in the polymer chain has made one of the most versatile materials Chitosan by the possibility to perform a wide variety of modifications such as reactions the anchor of enzymes for applications in biomedicine. [5]

(RN) retinol or vitamin A is fat-soluble and necessary is biological processes such as the formation and maintenance of epithelial cells. This vitamin is retinol, the retinal and Retinoic acid. Formed from provitamin beta carotene and other Pro-vitamins in the tract of the large intestine. It is stored in the liver. 
The most common natural polymers: silk, collagen, elastin, keratin and Chitosan are discussed as components of mixtures with artificial polymers. [6]

There are two main types of amino acids (AA) which are grouped in essential and non-essential. The essential AA can't be synthesized by the body and to obtain highprotein foods are consumed. The growth, repair and maintenance of cells depends on these amino acids. The AA is present in the body for skin regeneration: Leucine, interacting with isoleucine and valine to promote healing of muscle tissue, skin and bones.

\section{The ETC theory.}

The BG is defined as the energy difference between the valence band and the conduction band. In the BG there are no electronic states available; this means that when an electric field is applied, the electrons cannot increase their energy.

In quantum theory, it is known as HOMO and LUMO, and in the old theory they are known as E - and E +. The LUMO is defined as the range of electronic energy that allows acceleration in electrons by the presence of electrical currents and is also called conduction band; HOMO is defined as the highest energy interval that is occupied by electrons in absolute zero value and is called valence band. The HOMO is the most electron-filled orbital, while the LUMO is the orbital that lacks electrons. The HOMO equaled to zero (0 HOMO) is the last layer full of meaning that it is in the last orbital valence orbitals. The LUMO equaled to zero (0 LUMO) is the last layer that lacks electrons.

$\mathrm{EP}$ is defined as the total potential energy of the molecule. It is an electrostatic vector field that is defined as the potential that the electron needs to jump the Bohr radius (0.53 Armstrong) by its natural calculated electromotive force $(\mathrm{EMF})$. The negative E value (E-) is the electrostatic potential with negative poles, while the positive and value $(\mathrm{E}+)$ is the proton-electron potential [6]. The EP, in other words, means that having $1 \mathrm{EP}$ is having 1 volt for Armstrong. The EP is obtained by the absolute difference of $\mathrm{E}-$ and $\mathrm{E}+$.

The ETC is defined as the dimensionless parameter that describes an electrochemical reaction, which is interpreted as the number of times the potential energy needs to jump to the BG. It is calculated by dividing the BG and the EP entirely. That is, if it has a BG of 10 and an etc. of 40, it means that you need 40 times the EP value in $\mathrm{EV}$ so that the $\mathrm{BG}$ of 10 jumps from the HOMO to LUMO. [7, 8]

\section{MATERIALS AND METHODS}

SE-PM3 is a program for molecular modeling used by scientists to analyze the composition of molecules for quantum HOMO-LUMO, BG, EP and other properties.
These data are used to form the table where are the ETC's of the interaction between the Lev and the NB.

Table.1: Parameters used for quantum computing molecular orbitals HOMO and LUMO

\begin{tabular}{|l|c|l|l|}
\hline \multicolumn{1}{|c|}{ Parameter } & Value & \multicolumn{1}{|c|}{ Parameter } & \multicolumn{1}{|c|}{ Value } \\
\hline Total charge & 0 & Polarizability & Not \\
\hline $\begin{array}{l}\text { Spin } \\
\text { Multiplicity }\end{array}$ & 1 & $\begin{array}{l}\text { Geometry } \\
\text { Optimization } \\
\text { Algorithm }\end{array}$ & $\begin{array}{l}\text { Polak- } \\
\text { Ribière } \\
\text { (Conjugate } \\
\text { Gradient) }\end{array}$ \\
\hline Spin Pairing & RHF & $\begin{array}{l}\text { Termination } \\
\text { condition } \\
\text { RMS } \\
\text { gradient of }\end{array}$ & $\begin{array}{l}0.1 \\
\text { Kcal/Amol }\end{array}$ \\
\hline $\begin{array}{l}\text { State Lowest } \\
\text { Convergent } \\
\text { Limit }\end{array}$ & 0.01 & $\begin{array}{l}\text { Termination } \\
\text { condition or }\end{array}$ & $\begin{array}{l}\text { maximum } \\
1000 \text { cycles }\end{array}$ \\
\hline $\begin{array}{l}\text { Interaction } \\
\text { Limit }\end{array}$ & 50 & $\begin{array}{l}\text { Termination } \\
\text { condition or }\end{array}$ & In vacuo \\
\hline $\begin{array}{l}\text { Accelerate } \\
\text { Convergence }\end{array}$ & Yes & $\begin{array}{l}\text { Screen refresh } \\
\text { period }\end{array}$ & 1 cycle \\
\hline
\end{tabular}

The Software Hyperchem Professional performs Molecular modeling and analysis of the Lx, Qn and, Rl (Hyperchem, hypercube, Multi in for Windows, series 12800-1501800080)The font size for heading is 11 points bold face and subsections with 10 points and not bold. Do not underline any of the headings, or add dashes, colons, etc.

Table.2: Parameters used for access the map of the electrostatic potential of the molecules

\begin{tabular}{|l|l|l|l|}
\hline \multicolumn{1}{|c|}{ Parameter } & \multicolumn{1}{|c|}{ Value } & \multicolumn{1}{|c|}{ Parameter } & Value \\
\hline $\begin{array}{l}\text { Molecular } \\
\text { Property }\end{array}$ & $\begin{array}{l}\text { Property } \\
\text { Electrostatic } \\
\text { Potential }\end{array}$ & $\begin{array}{l}\text { Contour Grid } \\
\text { increment }\end{array}$ & 0.05 \\
\hline Representation & $\begin{array}{l}\text { 3D Mapped } \\
\text { Isosurface }\end{array}$ & $\begin{array}{l}\text { Mapped } \\
\text { Function } \\
\text { Options }\end{array}$ & Default \\
\hline $\begin{array}{l}\text { Isosurface } \\
\text { Grid: Grid } \\
\text { Mesh Size }\end{array}$ & Coarse & $\begin{array}{l}\text { Transparency } \\
\text { level }\end{array}$ & $\begin{array}{l}\text { A } \\
\text { criteria }\end{array}$ \\
\hline $\begin{array}{l}\text { Isosurface } \\
\text { Grid: Grid } \\
\text { Layout }\end{array}$ & Default & $\begin{array}{l}\text { Isosurface } \\
\text { Rendering: } \\
\text { Total charge } \\
\text { density } \\
\text { contour value }\end{array}$ & 0.015 \\
\hline $\begin{array}{l}\text { contour Grid: } \\
\text { Starting Value }\end{array}$ & Default & Rendering Wire Mesh \\
\hline
\end{tabular}




\section{RESULTS AND DISCUSSIONS}

Values are obtained from the simulation of molecules of the compounds in the software HYPERCHEM. The software calculates the bandgap, potential energy and energy transfer coefficient data (for its acronym in English, BG, the EP and the ETC). When designing the molecule gets the values of e- and e+ (HOMO-LUMO) in value to zero and a density of 0.015 . Cross compounds bands are taking the values of the initial calculation and swapping it with another compound interest. Under the cross-band ETC is the value of the more reactive compound.

Table.3: Distribution of values of three compounds to analyze

\begin{tabular}{|c|c|c|c|c|c|c|c|}
\hline $\begin{array}{l}\text { Su } \\
\text { bs }\end{array}$ & $\begin{array}{c}\text { HOM } \\
\text { O }\end{array}$ & $\begin{array}{c}\text { LUM } \\
\text { O }\end{array}$ & BG & E- & $\mathbf{E}+$ & EP & ETC \\
\hline Lx & $\begin{array}{c}- \\
9.351\end{array}$ & 0.278 & $\begin{array}{c}9.62 \\
9\end{array}$ & $\begin{array}{l}0.0 \\
03\end{array}$ & $\begin{array}{l}0.1 \\
04\end{array}$ & $\begin{array}{c}0 . \\
107\end{array}$ & $\begin{array}{c}89.9 \\
91\end{array}$ \\
\hline $\mathrm{Rl}$ & $\begin{array}{c}- \\
8.228\end{array}$ & $\begin{array}{c}- \\
0.483 \\
4\end{array}$ & $\begin{array}{c}7.74 \\
46\end{array}$ & $\begin{array}{c}0.1 \\
1\end{array}$ & $\begin{array}{l}0.1 \\
83\end{array}$ & $\begin{array}{c}0.2 \\
93\end{array}$ & $\begin{array}{c}26.4 \\
32\end{array}$ \\
\hline QN & $\begin{array}{c}- \\
9.726\end{array}$ & 1.662 & $\begin{array}{c}11.3 \\
88\end{array}$ & $\begin{array}{c}0.1 \\
14\end{array}$ & $\begin{array}{l}0.3 \\
28\end{array}$ & $\begin{array}{l}0.4 \\
42\end{array}$ & $\begin{array}{c}25.7 \\
64\end{array}$ \\
\hline
\end{tabular}

Table.4: Cross-band values

\begin{tabular}{|c|c|c|c|c|c|c|c|}
\hline $\begin{array}{l}\text { Su } \\
\text { bs }\end{array}$ & $\begin{array}{l}\text { HO } \\
\text { MO } \\
\end{array}$ & $\begin{array}{l}\text { LU } \\
\text { MO }\end{array}$ & BG & E- & E + & EP & $\begin{array}{l}\text { ET } \\
\text { C } \\
\end{array}$ \\
\hline $\begin{array}{l}\mathrm{Lx} \\
-\mathrm{Rl}\end{array}$ & -951 & $\begin{array}{l}- \\
0.483 \\
4\end{array}$ & $\begin{array}{l}8.86 \\
76\end{array}$ & $\begin{array}{l}- \\
0.0 \\
03\end{array}$ & $\begin{array}{l}0.1 \\
83\end{array}$ & 0.186 & $\begin{array}{l}47.6 \\
75\end{array}$ \\
\hline $\begin{array}{l}\text { Rl- } \\
\text { Lx }\end{array}$ & $\begin{array}{l}- \\
8.228\end{array}$ & 0.278 & $\begin{array}{l}8.50 \\
6\end{array}$ & $\begin{array}{l}0.1 \\
1\end{array}$ & $\begin{array}{l}0.1 \\
04\end{array}$ & 0.214 & $\begin{array}{l}39.7 \\
48\end{array}$ \\
\hline $\begin{array}{l}\text { Lx } \\
- \\
\text { Qn }\end{array}$ & $\begin{array}{l}- \\
9,351\end{array}$ & 1.662 & $\begin{array}{l}11.0 \\
13\end{array}$ & $\begin{array}{l}- \\
0.0 \\
03\end{array}$ & $\begin{array}{l}0.3 \\
28\end{array}$ & 0.331 & $\begin{array}{l}33.2 \\
72\end{array}$ \\
\hline $\begin{array}{l}\text { Qn } \\
- \\
\text { Lx }\end{array}$ & $\begin{array}{l}- \\
9.726\end{array}$ & 0.278 & $\begin{array}{l}10.0 \\
04\end{array}$ & $\begin{array}{l}0.1 \\
14\end{array}$ & $\begin{array}{l}0.1 \\
83\end{array}$ & 0.297 & $\begin{array}{l}33.6 \\
84\end{array}$ \\
\hline $\begin{array}{l}\text { Rl- } \\
\text { Qn }\end{array}$ & $-\overline{8.228}$ & 1.662 & 9.89 & $\begin{array}{l}- \\
0.1 \\
1\end{array}$ & $\begin{array}{l}0.3 \\
28\end{array}$ & 0.438 & $\begin{array}{l}22.5 \\
80\end{array}$ \\
\hline $\begin{array}{l}\text { Qn } \\
\text {-Rl }\end{array}$ & $\begin{array}{l}- \\
9.726\end{array}$ & $\begin{array}{l}0.483 \\
4\end{array}$ & $\begin{array}{l}9.24 \\
26\end{array}$ & $\begin{array}{l}0.1 \\
14\end{array}$ & $\begin{array}{l}0.1 \\
83\end{array}$ & $\begin{array}{l}0.297 \\
\text { (h) }\end{array}$ & $\begin{array}{l}31.1 \\
20\end{array}$ \\
\hline
\end{tabular}

The compounds work together to form a mixture homogeneous. Tables 3 and 4 show individual values and cross compounds to form the desired product. Displays the value of smaller ETC that these compounds produced the desired reaction.

Table.5: Values of pairs and trios of compounds

\begin{tabular}{|c|c|c|c|c|c|c|c|}
\hline Subs & $\begin{array}{l}\text { HO } \\
\text { MO }\end{array}$ & $\begin{array}{l}\text { LU } \\
\text { MO }\end{array}$ & BG & E- & $\mathbf{E}+$ & EP & $\begin{array}{l}\text { ET } \\
\text { C }\end{array}$ \\
\hline $\begin{array}{l}\text { LX- } \\
\text { RL }\end{array}$ & $\begin{array}{l}- \\
9,351\end{array}$ & $\begin{array}{l} \\
0.48 \\
34\end{array}$ & $\begin{array}{l}8.86 \\
76\end{array}$ & $\begin{array}{l}- \\
0.0 \\
03\end{array}$ & $\begin{array}{l}0.1 \\
83\end{array}$ & $\begin{array}{l}0.1 \\
86\end{array}$ & $\begin{array}{l}47.6 \\
75\end{array}$ \\
\hline $\begin{array}{l}\text { RL- } \\
\text { LX }\end{array}$ & $\begin{array}{l}- \\
8.228\end{array}$ & $\begin{array}{l}0.27 \\
8\end{array}$ & $\begin{array}{l}8.50 \\
6\end{array}$ & $\begin{array}{l}- \\
0.1 \\
1\end{array}$ & $\begin{array}{l}0.1 \\
04\end{array}$ & $\begin{array}{l}0.2 \\
14\end{array}$ & $\begin{array}{l}39.7 \\
48\end{array}$ \\
\hline $\begin{array}{l}\text { LX- } \\
\text { QN }\end{array}$ & $\overline{9}-351$ & $\begin{array}{l}1.66 \\
2\end{array}$ & $\begin{array}{l}11.0 \\
13\end{array}$ & $\begin{array}{l}- \\
0.0 \\
03\end{array}$ & $\begin{array}{l}0.3 \\
28\end{array}$ & $\begin{array}{l}0.3 \\
31\end{array}$ & $\begin{array}{l}33.2 \\
72\end{array}$ \\
\hline $\begin{array}{l}\text { QN- } \\
\text { LX }\end{array}$ & $\begin{array}{l}- \\
9.726\end{array}$ & $\begin{array}{l}0.27 \\
8\end{array}$ & $\begin{array}{l}10.0 \\
04\end{array}$ & $\begin{array}{l}0.1 \\
14\end{array}$ & $\begin{array}{l}0.1 \\
83\end{array}$ & $\begin{array}{l}0.2 \\
97\end{array}$ & $\begin{array}{l}33.6 \\
84\end{array}$ \\
\hline $\begin{array}{l}\text { RL- } \\
\text { QN }\end{array}$ & $\overline{8}-\overline{228}$ & $\begin{array}{l}1.66 \\
2\end{array}$ & 9.89 & $\begin{array}{l}- \\
0.1 \\
1\end{array}$ & $\begin{array}{l}0.3 \\
28\end{array}$ & $\begin{array}{l}0.4 \\
38\end{array}$ & $\begin{array}{l}22.5 \\
80\end{array}$ \\
\hline $\begin{array}{l}\text { QN- } \\
\text { RL }\end{array}$ & $\begin{array}{l}- \\
9.726\end{array}$ & $\begin{array}{l}0.48 \\
34\end{array}$ & $\begin{array}{l}9.24 \\
26\end{array}$ & $\begin{array}{l}- \\
0.1 \\
14\end{array}$ & $\begin{array}{l}0.1 \\
83\end{array}$ & $\begin{array}{l}0.2 \\
97\end{array}$ & $\begin{array}{l}31.1 \\
20\end{array}$ \\
\hline $\begin{array}{l}\text { QN- } \\
\text { RL/L } \\
\text { X }\end{array}$ & $\begin{array}{l}- \\
9.726\end{array}$ & $\begin{array}{l}0.27 \\
8\end{array}$ & $\begin{array}{l}10.0 \\
04\end{array}$ & $\begin{array}{l}- \\
0.1 \\
14\end{array}$ & $\begin{array}{l}0.1 \\
04\end{array}$ & $\begin{array}{l}0.2 \\
18\end{array}$ & $\begin{array}{l}45.8 \\
90\end{array}$ \\
\hline $\begin{array}{l}\text { LX/Q } \\
\text { N-RL }\end{array}$ & $\begin{array}{l}- \\
9,351\end{array}$ & $\begin{array}{l}0.48 \\
34\end{array}$ & $\begin{array}{l}8.86 \\
76\end{array}$ & $\begin{array}{l}- \\
0.0 \\
03\end{array}$ & $\begin{array}{l}0.1 \\
83\end{array}$ & $\begin{array}{l}0.1 \\
86\end{array}$ & $\begin{array}{l}47.6 \\
75\end{array}$ \\
\hline $\begin{array}{l}\text { RL- } \\
\text { QN/L } \\
\text { X } \\
\end{array}$ & $-\overline{8}$ & $\begin{array}{l}0.27 \\
8\end{array}$ & $\begin{array}{l}8.50 \\
6\end{array}$ & $\begin{array}{l}- \\
0.1 \\
1 \\
\end{array}$ & $\begin{array}{l}0.1 \\
04\end{array}$ & $\begin{array}{l}0.2 \\
14\end{array}$ & $\begin{array}{l}39.7 \\
48\end{array}$ \\
\hline $\begin{array}{l}\text { LX/R } \\
\text { L-QN }\end{array}$ & $\begin{array}{l}- \\
9.351\end{array}$ & $\begin{array}{l}1.66 \\
2\end{array}$ & $\begin{array}{l}11.0 \\
13\end{array}$ & $\begin{array}{l}- \\
0.0 \\
03\end{array}$ & $\begin{array}{l}0.3 \\
28\end{array}$ & $\begin{array}{l}0.3 \\
31\end{array}$ & $\begin{array}{l}33.2 \\
72\end{array}$ \\
\hline
\end{tabular}

Theoretical evidence for quantum analysis, show that the combination of compounds that interact with the AA (amino acids) that participate in the regeneration of epithelial tissue present in human skin and muscles is possible.

The $\mathrm{Lx}$ is the basis of this new biomaterial in combination with the substrates that held the interaction and the possible regeneration of epithelial tissue. Rl and Qn compounds have lower value ETC so that it concluded a high chemical reaction between the two.

Table 5 shows combinations of three compounds (Lx, Rl, Qn) for its combination with quantum chemistry obtaining a minor etc.

The Lx is the basis of this new biomaterial in combination with the substrates that held the interaction and the 
possible regeneration of epithelial tissue. Rl and Qn compounds have lower value ETC so that it concluded a high chemical reaction between the two.

Table.6: Interaction of reducing and oxidizing component of $L x$ and $R l$

\begin{tabular}{|l|l|l|l|l|l|}
\hline & $\begin{array}{l}\text { Reduc } \\
\text { er }\end{array}$ & $\begin{array}{l}\text { Oxidiz } \\
\text { er }\end{array}$ & $\begin{array}{l}\text { Interacti } \\
\text { on }\end{array}$ & $\begin{array}{l}\text { LTC } \\
\text { ts }\end{array}$ \\
\hline \multirow{2}{*}{$\begin{array}{l}\text { Pure } \\
\text { Substan } \\
\text { ces }\end{array}$} & LX & LX & LX - Lx & $\begin{array}{l}89.9 \\
90\end{array}$ & Top \\
\cline { 2 - 6 } & RL & RL & RL - Rl & $\begin{array}{l}26.4 \\
32\end{array}$ & $\begin{array}{l}\text { Low } \\
\text { er }\end{array}$ \\
\hline \multirow{2}{*}{$\begin{array}{l}\text { Cross } \\
\text { band }\end{array}$} & LX & RL & LX - R1 & $\begin{array}{l}41.6 \\
31\end{array}$ & \\
\cline { 2 - 5 } & RL & LX & RL - Lx & $\begin{array}{l}39.5 \\
62\end{array}$ \\
\hline
\end{tabular}

Graphs of quantum well (figures 1) have two lines that represent the upper and lower limits (greater ETC). Black dots plotted are the percentage of probability of occurring a chemical reaction with the combination of two of the three compounds.

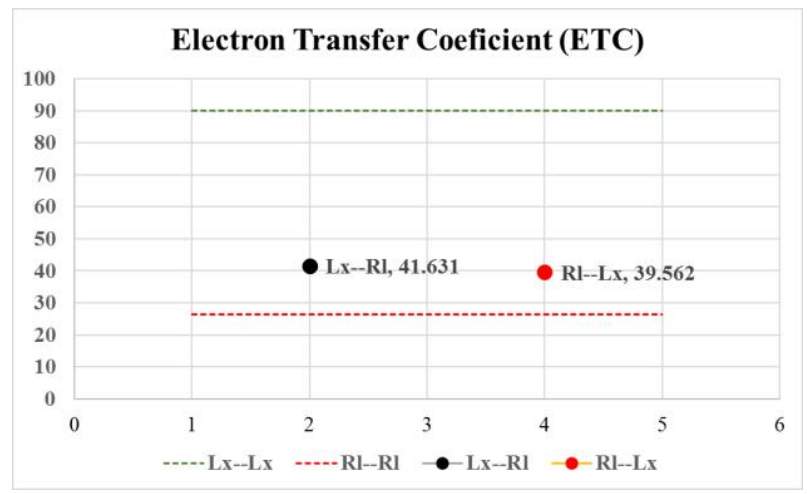

Fig.1: Quantum well, Lx-Rl ETC interaction

Tables 6 and 7 show interactions between reducing and oxidizing compounds for the upper and lower limits and graphing quantum wells etc.

Table.7: Interaction of the component reducing and oxidizing of Lx and Qn

\begin{tabular}{|l|l|l|l|l|l|}
\hline & $\begin{array}{l}\text { Reduc } \\
\text { er }\end{array}$ & $\begin{array}{l}\text { Oxidiz } \\
\text { er }\end{array}$ & $\begin{array}{l}\text { Interacti } \\
\text { on }\end{array}$ & ETC & $\begin{array}{l}\text { Limi } \\
\text { ts }\end{array}$ \\
\hline \multirow{2}{*}{$\begin{array}{l}\text { Pure } \\
\text { Substan } \\
\text { ces }\end{array}$} & LX & LX & LX - Lx & $\begin{array}{l}89.9 \\
9\end{array}$ & Top \\
\cline { 2 - 5 } & Qn & Qn & Qn - Qn & $\begin{array}{l}26.4 \\
32\end{array}$ & $\begin{array}{l}\text { Low } \\
\text { er }\end{array}$ \\
\hline \multirow{2}{*}{$\begin{array}{l}\text { Cross } \\
\text { band }\end{array}$} & LX & Qn & LX - Qn & $\begin{array}{l}33.2 \\
71\end{array}$ & \\
\cline { 2 - 5 } & Qn & LX & Qn - Lx & $\begin{array}{l}33.6 \\
83\end{array}$ & \\
\hline
\end{tabular}

Figure 1 shows an average probability of interaction between the two compounds (50\% probability). Figure 2 shows the same probability of interaction.

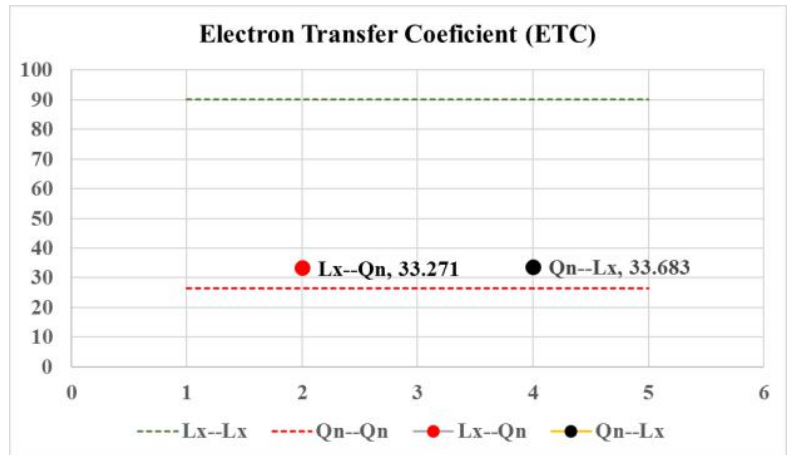

Fig.2: Quantum well, the interaction between $L x$ and $Q n$ ETC
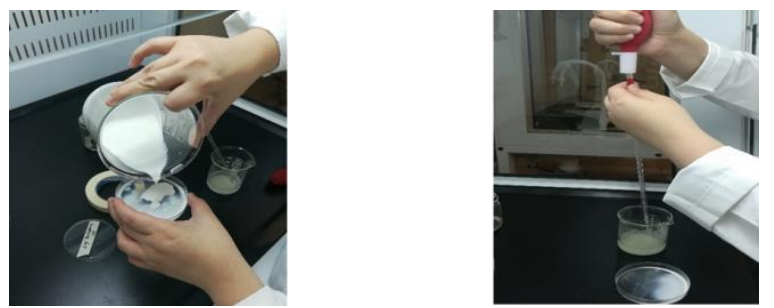

Fig.3: Emptying of Lx, Qn and Rl for field testing on lab in the formation of a new biomaterial

In Figure 3 are observed practices carried out in the laboratory to check the quantum theoretical calculated previously.
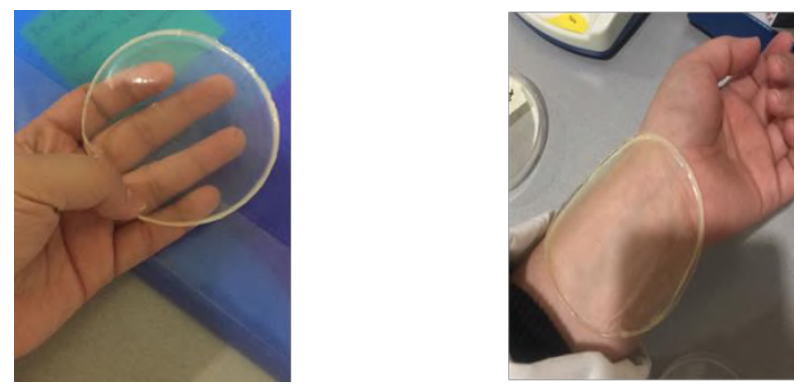

Fig.4: Form of Biomaterial with Lx, Qn, and Rl

Figure 4 shows laboratory tests proving that quantum calculation (calculation of high chemical reaction between compounds) ETC is the biomaterial.

Table 8 shows the interactions of mixtures of the biomaterial the AA main compounds necessary for the regeneration of epithelial and muscle tissue. Minor interactions are likely chemical reaction between the mixtures working as oxidants and antioxidants. Obtaining ETC describes that the reaction will be likely to benefit from the growth of epithelial cells in patients who have superficial wounds. 
Table.8: Pairs of AA with mixtures of $L x, Q n$, and $R l$

\begin{tabular}{|c|c|c|c|c|c|c|c|c|c|}
\hline $\begin{array}{l}\mathbf{N} \\
\mathbf{0}\end{array}$ & $\begin{array}{l}\text { Red } \\
\text { ucer }\end{array}$ & $\begin{array}{c}\text { Oxi } \\
\text { dize } \\
\mathbf{r}\end{array}$ & $\begin{array}{l}\text { HO } \\
\text { MO }\end{array}$ & $\begin{array}{l}\text { LU } \\
\text { MO }\end{array}$ & $\begin{array}{l}\mathbf{B} \\
\mathbf{G}\end{array}$ & $\begin{array}{l}\mathbf{E} \\
-\end{array}$ & $\begin{array}{l}\mathbf{E} \\
+\end{array}$ & $\begin{array}{l}\mathbf{E} \\
\mathbf{P}\end{array}$ & $\begin{array}{l}\mathbf{E} \\
\mathbf{T} \\
\mathbf{C}\end{array}$ \\
\hline 1 & $\begin{array}{l}\text { QN- } \\
\text { RL/ } \\
\text { LX }\end{array}$ & $\begin{array}{c}\text { LEU } \\
- \\
\text { GLY }\end{array}$ & $\begin{array}{c}- \\
9.73\end{array}$ & $\begin{array}{c}0.9 \\
0\end{array}$ & $\begin{array}{l}10 . \\
63\end{array}$ & $\begin{array}{l}0 . \\
1 \\
1\end{array}$ & $\begin{array}{l}0 . \\
1 \\
6\end{array}$ & $\begin{array}{l}0 . \\
2 \\
7\end{array}$ & $\begin{array}{c}38 \\
.9\end{array}$ \\
\hline 2 & $\begin{array}{l}\text { QN- } \\
\text { RL/ } \\
\text { LX }\end{array}$ & $\begin{array}{c}\text { LEU } \\
- \\
\text { LYS }\end{array}$ & $\begin{array}{c}- \\
9.73\end{array}$ & $\begin{array}{c}0.9 \\
4\end{array}$ & $\begin{array}{l}10 . \\
67\end{array}$ & $\begin{array}{l}0 . \\
1 \\
1\end{array}$ & $\begin{array}{l}0 . \\
1 \\
9\end{array}$ & $\begin{array}{l}0 . \\
3 \\
1\end{array}$ & $\begin{array}{c}34 \\
.5\end{array}$ \\
\hline 3 & $\begin{array}{c}\text { LEU } \\
- \\
\text { LYS }\end{array}$ & $\begin{array}{l}\text { QN- } \\
\text { RL/ } \\
\text { LX }\end{array}$ & $\begin{array}{c}- \\
9.64\end{array}$ & $\begin{array}{c}0.2 \\
7\end{array}$ & $\begin{array}{c}9.9 \\
2\end{array}$ & $\begin{array}{l}0 . \\
1 \\
3\end{array}$ & $\begin{array}{l}0 . \\
1 \\
0\end{array}$ & $\begin{array}{l}0 . \\
2 \\
3\end{array}$ & $\begin{array}{c}43 \\
.1\end{array}$ \\
\hline 4 & $\begin{array}{l}\text { QN- } \\
\text { RL/ } \\
\text { LX }\end{array}$ & $\begin{array}{l}\text { ILE- } \\
\text { GLN }\end{array}$ & $\begin{array}{c}- \\
9.73\end{array}$ & $\begin{array}{c}0.7 \\
5\end{array}$ & $\begin{array}{l}10 . \\
48\end{array}$ & $\begin{array}{l}0 . \\
1 \\
1\end{array}$ & $\begin{array}{l}0 . \\
1 \\
9\end{array}$ & $\begin{array}{l}0 . \\
3 \\
1\end{array}$ & $\begin{array}{c}34 \\
.2\end{array}$ \\
\hline 5 & $\begin{array}{l}\text { QN- } \\
\text { RL/ } \\
\text { LX }\end{array}$ & $\begin{array}{l}\text { ILE- } \\
\text { LYS }\end{array}$ & $\begin{array}{c}- \\
9.73\end{array}$ & $\begin{array}{c}0.9 \\
4\end{array}$ & $\begin{array}{l}10 . \\
67\end{array}$ & $\begin{array}{l}0 . \\
1 \\
1\end{array}$ & $\begin{array}{l}0 . \\
1 \\
9\end{array}$ & $\begin{array}{l}0 . \\
3 \\
1\end{array}$ & $\begin{array}{l}34 \\
.5\end{array}$ \\
\hline 6 & $\begin{array}{l}\text { QN- } \\
\text { RL/ } \\
\text { LX }\end{array}$ & $\begin{array}{l}\text { GLY } \\
\text {-ILE }\end{array}$ & $\begin{array}{c}- \\
9.73\end{array}$ & $\begin{array}{c}0.9 \\
7\end{array}$ & $\begin{array}{l}10 . \\
70\end{array}$ & $\begin{array}{c}- \\
0 . \\
1 \\
1\end{array}$ & $\begin{array}{l}0 . \\
1 \\
9\end{array}$ & $\begin{array}{l}0 . \\
3 \\
0\end{array}$ & $\begin{array}{l}35 \\
.4\end{array}$ \\
\hline 7 & $\begin{array}{l}\text { QN- } \\
\text { RL/ } \\
\text { LX }\end{array}$ & $\begin{array}{c}\text { GLY } \\
- \\
\text { GLN }\end{array}$ & $\begin{array}{c}- \\
9.73\end{array}$ & $\begin{array}{c}0.7 \\
5\end{array}$ & $\begin{array}{l}10 . \\
48\end{array}$ & $\begin{array}{l}0 . \\
1 \\
1\end{array}$ & $\begin{array}{l}0 . \\
1 \\
9\end{array}$ & $\begin{array}{l}0 . \\
3 \\
1\end{array}$ & $\begin{array}{c}34 \\
.2\end{array}$ \\
\hline 8 & $\begin{array}{l}\text { QN- } \\
\text { RL/ } \\
\text { LX }\end{array}$ & $\begin{array}{l}\text { LYS } \\
\text {-ILE }\end{array}$ & $\begin{array}{c}- \\
9.73\end{array}$ & $\begin{array}{c}0.9 \\
7\end{array}$ & $\begin{array}{l}10 . \\
70\end{array}$ & $\begin{array}{l}- \\
0 . \\
1 \\
1\end{array}$ & $\begin{array}{l}0 . \\
1 \\
9\end{array}$ & $\begin{array}{l}0 . \\
3 \\
0\end{array}$ & $\begin{array}{l}35 \\
.4\end{array}$ \\
\hline 9 & $\begin{array}{l}\text { QN- } \\
\text { RL/ } \\
\text { LX }\end{array}$ & $\begin{array}{c}\text { VAL } \\
- \\
\text { GLN }\end{array}$ & $\begin{array}{c}- \\
9.73\end{array}$ & $\begin{array}{c}0.7 \\
5\end{array}$ & $\begin{array}{l}10 . \\
48\end{array}$ & $\begin{array}{c}- \\
0 . \\
1 \\
1\end{array}$ & $\begin{array}{l}0 . \\
1 \\
9\end{array}$ & $\begin{array}{l}0 . \\
3 \\
1\end{array}$ & $\begin{array}{c}34 \\
.2\end{array}$ \\
\hline $\begin{array}{l}1 \\
0\end{array}$ & $\begin{array}{l}\text { QN- } \\
\text { RL/ } \\
\text { LX }\end{array}$ & $\begin{array}{l}\text { VAL } \\
\text {-ILE }\end{array}$ & $\begin{array}{c}- \\
9.73\end{array}$ & $\begin{array}{c}0.9 \\
7\end{array}$ & $\begin{array}{l}10 . \\
70\end{array}$ & $\begin{array}{l}- \\
0 . \\
1 \\
1\end{array}$ & $\begin{array}{l}0 . \\
1 \\
9\end{array}$ & $\begin{array}{l}0 . \\
3 \\
0\end{array}$ & $\begin{array}{l}35 \\
.4\end{array}$ \\
\hline $\begin{array}{l}1 \\
1\end{array}$ & $\begin{array}{l}\text { QN- } \\
\text { RL/ } \\
\text { LX }\end{array}$ & $\begin{array}{c}\text { VAL } \\
- \\
\text { LYS }\end{array}$ & $\begin{array}{c}- \\
9.73\end{array}$ & $\begin{array}{c}0.9 \\
4\end{array}$ & $\begin{array}{c}10 . \\
67\end{array}$ & $\begin{array}{l}- \\
0 . \\
1 \\
1\end{array}$ & $\begin{array}{l}0 . \\
1 \\
9\end{array}$ & $\begin{array}{l}0 . \\
3 \\
1\end{array}$ & $\begin{array}{c}34 \\
.5\end{array}$ \\
\hline $\begin{array}{l}1 \\
2\end{array}$ & $\begin{array}{c}\text { LEU } \\
- \\
\text { GL } \\
N\end{array}$ & $\begin{array}{l}\text { LX/ } \\
\text { RL- } \\
\text { QN }\end{array}$ & $\begin{array}{c}- \\
9.64\end{array}$ & $\begin{array}{c}1.6 \\
6\end{array}$ & $\begin{array}{l}11 . \\
31\end{array}$ & $\begin{array}{l}0 . \\
1 \\
3\end{array}$ & $\begin{array}{l}0 . \\
3 \\
3\end{array}$ & $\begin{array}{l}0 . \\
4 \\
5\end{array}$ & $\begin{array}{c}24 \\
.9\end{array}$ \\
\hline
\end{tabular}

\begin{tabular}{|c|c|c|c|c|c|c|c|c|c|}
\hline 1 & $\begin{array}{c}\text { LEU } \\
- \\
\text { GL } \\
\text { Y }\end{array}$ & $\begin{array}{l}\text { LX/ } \\
\text { RL- } \\
\text { QN }\end{array}$ & $\begin{array}{c}- \\
9.64\end{array}$ & $\begin{array}{c}1.6 \\
6\end{array}$ & $\begin{array}{l}11 . \\
31\end{array}$ & $\begin{array}{c}- \\
0 . \\
1 \\
3\end{array}$ & $\begin{array}{l}0 . \\
3 \\
3\end{array}$ & $\begin{array}{l}0 . \\
4 \\
5\end{array}$ & $\begin{array}{c}24 \\
.9\end{array}$ \\
\hline $\begin{array}{l}1 \\
4\end{array}$ & $\begin{array}{c}\text { LEU } \\
- \\
\text { LYS }\end{array}$ & $\begin{array}{l}\text { LX/ } \\
\text { RL- } \\
\text { QN }\end{array}$ & $\begin{array}{c}- \\
9.64\end{array}$ & $\begin{array}{c}1.6 \\
6\end{array}$ & $\begin{array}{l}11 . \\
31\end{array}$ & $\begin{array}{c}- \\
0 . \\
1 \\
3\end{array}$ & $\begin{array}{l}0 . \\
3 \\
3\end{array}$ & $\begin{array}{l}0 . \\
4 \\
5\end{array}$ & $\begin{array}{r}24 \\
.9\end{array}$ \\
\hline $\begin{array}{l}1 \\
5\end{array}$ & $\begin{array}{l}\text { ILE- } \\
\text { LYS }\end{array}$ & $\begin{array}{l}\text { LX/ } \\
\text { RL- } \\
\text { QN }\end{array}$ & $\begin{array}{c}- \\
9.87\end{array}$ & $\begin{array}{c}1.6 \\
6\end{array}$ & $\begin{array}{l}11 . \\
53\end{array}$ & $\begin{array}{c}0 . \\
1 \\
3\end{array}$ & $\begin{array}{l}0 . \\
3 \\
3\end{array}$ & $\begin{array}{l}0 . \\
4 \\
5\end{array}$ & $\begin{array}{r}25 \\
.1\end{array}$ \\
\hline $\begin{array}{l}1 \\
6\end{array}$ & $\begin{array}{c}\text { GL } \\
\text { Y- } \\
\text { ILE }\end{array}$ & $\begin{array}{l}\text { LX/ } \\
\text { RL- } \\
\text { QN }\end{array}$ & $\begin{array}{c}- \\
9.90\end{array}$ & $\begin{array}{c}1.6 \\
6\end{array}$ & $\begin{array}{l}11 . \\
56\end{array}$ & $\begin{array}{c}- \\
0 . \\
1 \\
4\end{array}$ & $\begin{array}{l}0 . \\
3 \\
3\end{array}$ & $\begin{array}{l}0 . \\
4 \\
6\end{array}$ & $\begin{array}{c}24 \\
.9\end{array}$ \\
\hline $\begin{array}{l}1 \\
7\end{array}$ & $\begin{array}{c}\text { LYS } \\
- \\
\text { GL } \\
\text { Y }\end{array}$ & $\begin{array}{l}\text { LX/ } \\
\text { RL- } \\
\text { QN }\end{array}$ & $\begin{array}{c}- \\
9.52\end{array}$ & $\begin{array}{c}1.6 \\
6\end{array}$ & $\begin{array}{c}11 . \\
18\end{array}$ & $\begin{array}{c}- \\
0 . \\
1 \\
3\end{array}$ & $\begin{array}{l}0 . \\
3 \\
3\end{array}$ & $\begin{array}{l}0 . \\
4 \\
5\end{array}$ & $\begin{array}{l}24 \\
.6\end{array}$ \\
\hline $\begin{array}{l}1 \\
8\end{array}$ & $\begin{array}{c}\text { VA } \\
\text { L- } \\
\text { GL } \\
\text { N }\end{array}$ & $\begin{array}{l}\text { LX/ } \\
\text { RL- } \\
\text { QN }\end{array}$ & $\begin{array}{c}- \\
9.91\end{array}$ & $\begin{array}{c}1.6 \\
6\end{array}$ & $\begin{array}{l}11 . \\
58\end{array}$ & $\begin{array}{c}- \\
0 . \\
1 \\
3\end{array}$ & $\begin{array}{l}0 . \\
3 \\
3\end{array}$ & $\begin{array}{l}0 . \\
4 \\
6\end{array}$ & $\begin{array}{r}25 \\
.2\end{array}$ \\
\hline
\end{tabular}

\section{CONCLUSION}

1. The Lx is highly reaccionable with compounds Qn and R1.

2. The combination of the three compounds (Lx, Qn, $\mathrm{Rl}$ ) is possible by low levels that present etc.

3. The highest interaction is with the Qn and Rl when working as antioxidant and oxidant respectively.

4. By combining three compounds is calculated an etc. of 33.2719033 when the Lx works as a reducing agent.

5. The compounds shown in its quantum wells High solubility and formation of striation.

6. Experiments prove the quantum chemistry calculations.

7. Forms a patch of Lx, Qn, and R1.

8. The ETC child occur with Rl-Qn 22.57.

9. Lx, Qn, and Rl compounds interact with the AA of epithelial and muscle tissue.

10. The combination of the three main compounds for the biomaterial have high reaction with the AA.

\section{REFERENCES}

[1] Narciso, A. R. M. (1974). The exploitation of rubber in Mexico and its use in the world.

[2] Carreon Blaine, Emilie a. (2006). The olli in the plastic Aztec: the use of rubber in the 16th century. UNAM. ISBN 970-32-2200-5. 
[3] Fireman, Philip. Atlas of Allergy and Immunology clinic. Elsevier Mosby. p-261.

[4] Rosa, S. D. S. R. F., do Carmo Reis, M., Rosa, M. F. F., Colon, D., dos Reis, C. A., \& Balthazar, J. M. (2015). Use of Natural Latex as a Biomaterial for the Treatment of Diabetic Foot-A New Approach to Treating Symptoms of Diabetes Mellitus. In Topics in Public Health. InTech.

[5] Velasquez, C. (2003). Some applications of chitosan in aqueous systems. Revista Iberoamericana of polymers, 4 (2), 91.

[6] Sionkowska, a. (2011). Current research on the blends of natural and synthetic polymers as new biomaterials. Progress in Polymer Science, 36 (9): 1254-1276.

[7] Mondragon-jimenez, jesus francisco, et al. interaction of methylenedioxymethamphetamine vs. neurotransmitters and the relationship by the quantum method. 2017

[8] Gonzalez-perez, m. chemical-quantum analysis of the aggressiveness of glucose and its appeasement with atp inside the cell and water as an excellent antioxidant, 2017.

[9] Firmin H. Aikpo, Miriac Dimitri S. Ahouanse, Lucien Agbandji, Patrick A. Edorh, Christophe S. Houssou(2017).Assessment of contamination of soil by pesticides in Djidja's cotton area in Benin. International Journal of Advanced Engineering Research and Science (ISSN : 2349-6495(P) | 24561908(O)),4(7), 001-005. http://dx.doi.org/10.22161/ijaers.4.7.1

[10] Perfect, T. J., \& Schwartz, B. L. (Eds.) (2002). Applied metacognition Retrieved from http://www.questia.com/read/107598848

[11] Myers, D. G. (2007). Psychology(1stCanadian ed.). New York, NY: Worth.

[12] Cognition.(2008). In Oxford reference online premium dictionary. Retrieved from http://www.oxfordreference.com

[13]Blue, L. (2008, March 12).Is our happiness preordained? [Online exclusive]. Time. Retrieved from http://www.time.com/time/health

[14] J. Clerk Maxwell, A Treatise on Electricity and Magnetism, 3rd ed., vol. 2. Oxford: Clarendon, 1892, pp.68-73.

[15] I. S. Jacobs and C. P. Bean, "Fine particles, thin films and exchange anisotropy," in Magnetism, vol. III, G. T. Rado and H. Suhl, Eds. New York: Academic, 1963, pp. 271-350.

[16] K. Elissa, "Title of paper if known," unpublished.

[17]R. Nicole, "Title of paper with only first word capitalized," J. Name Stand. Abbrev., in press. 